\title{
Reflexiones sobre la Ley 34/2003 (1). Consideraciones generales sobre las tablas de indemnización de daños personales.
}

\author{
Reflections on Law 34/2003 (1). General considerations \\ on the indemnization tables for personal damage.
}

\author{
PM. Garamendi ${ }^{1}$
}

\section{RESUMEN}

El 5 de noviembre de 2003 se publicó la nueva Ley 34/2003 de modificación y adaptación a la normativa comunitaria de la legislación de seguros privados que incluyó la modificación de la tabla VI de baremo de indemnización de secuelas definitivas de la Ley 30/1995 de ordenación y supervisión de seguros privados. En nuestro país, el citado baremo ha constituido y constituye la herramienta aplicada en el contexto judicial para la indemnización económica de las lesiones producidas con motivo de accidentes de tráfico. La anterior redacción del citado baremo ha sido motivo de enconadas controversias en los distintos foros de debate relacionados con la Valoración del Daño Corporal desde su implantación. Sin negar la necesidad de algún instrumento de valoración común que colaborase a evitar la situación de caos aparente existente en nuestro país hasta la implantación del baremo de tráfico, han sido múltiples las críticas expresadas sobre su fundamento científico, validez y redacción final. El presente trabajo intenta adelantar algunas críticas y propuestas de mejora sobre la nueva redacción del baremo.

Palabras clave: accidentes de tráfico; baremos; ley 34/2003; medicina basada en la evidencia; valoración del daño corporal.

\section{ABSTRACT}

On November 52003 the new Law 34/2003 of modification and adaptation to the community normative of the legislation of private insurance which included the modification of chart $\mathrm{VI}$ of the scale of compensation of definitive sequels of the Law 30/1995 of ordination and supervision of private insurance was published. In our country, the mentioned scale has constituted and constitutes the tool applied in the judicial context for the economic compensation of the lesions taken place on the occasion of traffic accidents. The previous writing of the mentioned scale has been from its beginning the motive inflamed controversies in the different debate forums related with the Valuation of Corporal Damage. Without denying the necessity of some instrument of common valuation to collaborate in avoiding the situation of existent apparent chaos in our country until the installation of the traffic scale, there has been much criticism expressed on their scientific foundation, validity and final expression. The present work tries to advance some criticism and proposals for improvement on the new composition of the scale.

Key words: traffic accidents; guides for the assessment; Spanish Law 34/2003; evidence based medicine; permanent impairment assessment.

Correspondencia: Pedro Manuel Garamendi González. Servicio de Clínica Médico Forense. c/ Buenos Aires, nº 6 , planta 4. 48001 Bilbao. garamendi.pm@aju.ej-gv.es.

${ }^{1}$ Médico Forense. Especialista en Medicina Legal y Forense. IVML. Vizcaya. 


\section{INTRODUCCIÓN:}

El 5 de noviembre de 2003 se publicó la nueva Ley 34/2003 de modificación y adaptación a la normativa comunitaria de la legislación de seguros privados [I] que incluyó la modificación de la tabla VI de baremo de indemnización de secuelas definitivas de la Ley 30/1995 de ordenación y supervisión de seguros privados [2]. En nuestro país, el citado baremo ha constituido y constituye la herramienta aplicada en el contexto judicial para la indemnización económica de las lesiones producidas con motivo de accidentes de tráfico.

La anterior redacción del baremo, incluida en la tabla VI de la Ley 30/I995, fue motivo de enconada controversia desde su implantación en nuestro país. Dicha tabla fue una respuesta lógica a la situación de caos indemnizatorio por secuelas en accidentes de tráfico existente en nuestro país hasta entonces [3]. Tanto desde los foros médico legales, como desde las compañías aseguradoras y desde los propios medios judiciales [3], la medida fue aplaudida como un medio de unificación y armonización de una situación difícilmente sostenible antes de 1995 en relación con la indemnización de daños personales en accidentes de tráfico. Sin embargo, pese a ello, en los 8 años de vida efectiva del baremo, este ha sido objeto de múltiples críticas desde los distintos colectivos vinculados a su uso habitual. Las críticas principales fueron centradas en su redacción imprecisa, fundamento científico altamente dudoso y terminología no actualizada al actual estado de conocimientos en ciencias biomédicas [4].

La nueva redacción de la tabla-baremo de indemnización de daños personales ha sido presentada por sus autores en distintos foros nacionales como una mejora significativa en relación con su precedente. Se han argumentado por parte de los mismos mejoras en la ordenación y jerarquización de las secuelas, en el fundamento científico de la cuantificación del daño corporal, en el equilibrio en las cuantías de indemnización entre distintos capítulos del baremo y en el ajuste terminológico a la terminología médica más actualizada $[5,6]$. Sin embargo, pese a reconocer las mejoras evidentes en la redacción de la nueva tabla baremo y los esfuerzos por parte del grupo de trabajo auspiciado por UNESPA, el texto final presentado a las Cámaras Legislativas en junio de 2003 y el texto definitivo publicado en el BOE en noviembre de 2003 están aún lejos de constituir un instrumento idóneo de indemnización de daños personales $[7,8]$.

\section{ORÍGENES Y ANTECEDENTES:}

No es posible intentar entender la magnitud de la necesidad de un adecuado sistema de indemnización de daños corporales sin realizar algunas reflexiones sobre el contexto jurídico en el que el mismo se enmarca en nuestro ordenamiento nacional y en el ordenamiento de otros países de nuestro entorno [3].

En líneas generales, a nivel internacional se describen dos grandes tipos de sistemas legales en relación con la reparación de los daños corporales: los sistemas cerrados o de tipicidad y los sistemas abiertos o de atipicidad del ilícito, entre los cuales se cuenta el sistema español. Ejemplos clásicos del sistema cerrado los constituyen el derecho alemán y anglosajón (Common Law), mientras que son ejemplos del sistema abierto los del derecho francés e italiano. Si bien las diferencias entre ambos sistemas eran originariamente marcadas, el desarrollo actual ha hecho que las diferencias efectivas entre los distintos sistemas se hayan desdibujado, de modo que en la actualidad es difícil establecer unos límites teóricos marcados entre los distintos sistemas legales.

El derecho en los sistemas cerrados determina que únicamente se considera como daño jurídicamente relevante aquel que recae en la esfera de determinados derechos "absolutos". De este modo, los supuestos de daños reparables están tasados de forma aparentemente inflexible por la 
Ley. En la práctica, tampoco se exige que la ley deba contener todas las hipótesis que pueden dar lugar a responsabilidad de forma expresa, sino que es suficiente que se aludan con carácter general las hipótesis a las cuales la ley confiere el particular carácter de reparables por el tipo de interés lesionado.

En este contexto, el sistema anglosajón establece en el caso de la reparación de los daños personales la clara distinción entre los llamados special damages o pre-trial damages o daños económicos y los daños no económicos o general damages.

Los primeros (daños económicos) conforman los daños económicos probados antes de la fecha del juicio. Se incluyen en ellos tanto las pérdidas en los salarios que se han dejado de percibir, como los gastos realizados con motivo del accidente (viajes al hospital, medicamentos, costos del tratamiento médico privado o público, gastos por adaptación de la vivienda, gastos por la contratación de una persona que cuide al enfermo).

Los daños no económicos se clasifican en los tribunales ingleses en:

- Daños por el sufrimiento y el dolor: en ellos se agrupan todos los daños físicos y psíquicos pasados o futuros consecutivos al daño personal.

- Daños de relación por pérdida de placeres (loss of amenities): perjuicio moral por la imposibilidad de practicar actividades gratificantes para el lesionado que constituían sus hobbies.

- Daños por pérdida de ganancias futuras (future earnings): para calcular éstas, se toma en consideración la renta anual del lesionado, sus posibilidades de promoción profesional y se multiplican por el número de años que se prevé que el lesionado se hallará en situación de incapacidad. Cuando se supone que será una situación irreversible, se hace una valoración de la expectativa de vida.

En general el sistema anglosajón no supone diferencias teóricas sustanciales con los sistemas abiertos. En definitiva, busca, como el resto, la reparación completa de los daños personales. Lo que diferencia fundamentalmente al sistema británico del resto de sistemas es su distinto talante ante la indemnización de unos y otros tipos de daños. Los daños económicos cuantificables son reparados por los tribunales sin restricciones y en general los tribunales son abiertos a la hora de establecer indemnizaciones por estos daños, procurando resarcir todas las categorías reclamadas. Sin embargo, los daños puramente morales son valorados de forma más arbitraria y, en el fondo, restrictiva. Los tribunales se han mostrado francamente reacios a valorar los daños provocados por el sufrimiento y el dolor. Del mismo modo, los daños morales indirectos a los familiares, en concepto de pena y sufrimiento por la muerte de un familiar, son indemnizados con una cantidad económica tasada pero no se amplían por posibles pérdidas de ganancia de los familiares dependientes del fallecido, al considerar que ya son reparados con la reparación de los daños económicos del lesionado.

En resumen, el sistema anglosajón supone un sistema de mayor seguridad jurídica ya que establece un carácter tasado en sus indemnizaciones y evita posibles especulaciones sobre las peticiones de reparación económica. De otra parte, resulta un sistema uniforme en el que todos los capítulos del daño personal, con independencia de su origen en unos u otros tipos de actos ilícitos son reparados de forma uniforme. Sin embargo, resulta restrictivo sobre el aspecto relativo a los daños puramente morales al establecer reparaciones económicas según cuantías tasadas de forma uniforme. No obstante, esta actitud resulta dudosamente discutible si consideramos que en todo caso la reparación económica de los daños morales siempre resultará de alguna manera arbitraria. 
Por oposición, en los sistemas abiertos, las normas de responsabilidad civil extracontractual no limitan a priori los supuestos de daños reparables. En estos sistemas se permite al intérprete del Derecho un amplio margen de discrecionalidad para configurar las líneas o el marco de responsabilidad civil extracontractual en las que late el principio de alterum non laedere.

En estos sistemas, la apreciación de la cuantía y de las modalidades de indemnización de los daños es más abierta que en los sistemas cerrados. En Francia, esta apertura provoca problemas a la hora de establecer la cuantías de reparación de daños económicos. Sin embargo, en relación con los daños morales es en este país donde se han planteado mayores posibilidades de reparación, estableciendo reparaciones independientes por los distintos aspectos del daño moral diferenciadas según el régimen de responsabilidad civil aplicable al caso.

No obstante, los mayores problemas de los sistemas abiertos residen en dos áreas principales que se destacan también en el sistema español. De una parte, se aprecia la existencia de una pluralidad de regímenes de responsabilidad civil que dan lugar a grandes desigualdades entre víctimas con iguales dolencias físicas en función de las condiciones en las que se produzca el hecho ilícito. Así, resulta previsible que, en el caso de un accidente el responsable tenga un seguro de responsabilidad civil, las cuantías de la reparación sean mucho mayores que en el supuesto de una responsable sin seguro. En el extremo opuesto, la reparación de daños en el caso de un accidente en el que sea aplicable el seguro obligatorio de viajeros será muy inferior a la eventualmente obtenida por la aplicación de la legislación general de seguros por accidentes de tráfico.

El segundo gran problema de los sistemas abiertos y de parte de los sistemas cerrados, reside en la trascendencia económica que las reparaciones de los daños personales condicionan en la economía general de los países. El incremento generalizado de las indemnizaciones ha provocado un aumento progresivo de las primas de seguros. En EE.UU., las compañías aseguradoras se muestran reticentes a asegurar los daños producidos por negligencias profesionales médicas y en España existen dificultades para obtener seguros de responsabilidad para motoristas o conductores noveles en algunas compañías. Este segundo problema ha impulsado a autores como Tunc [3] durante años a recomendar que los daños derivados del pretium doloris y del daño estético no sean indemnizados para evitar una escalada indemnizatoria incontrolable. En el fondo, la reticencia general de la jurisprudencia anglosajona sobre la reparación del daño moral por dolor y sufrimiento responde a esta recomendación general de los analistas.

\section{ANÁLISIS PRELIMINAR DEL SISTEMA DE BAREMO DE LA LEY 34/2003:}

El Proyecto de la Ley 34/2003 tuvo su entrada en el Congreso de los Diputados el 30-62003 [9]. Tras el estudio del mismo, en sesión extraordinaria de 8-7-03 se discutió el proyecto, así como la enmienda a la totalidad propuesta por el Grupo Socialista y las enmiendas propuestas por los grupos Popular y Catalán. El grupo federal de Izquierda Unida presentó una enmienda de suspensión que no fue estimada por la Cámara.

En particular, el análisis planteado por el Grupo Socialista encaró el asunto apuntando el hecho de que había una desaparición masiva e injustificada de categorías de secuelas indemnizables y una evidente disminución global de puntuaciones, lo que redundaba en una disminución de los pagos que se realizarían a los damnificados mediante la aplicación del nuevo baremo frente al existente (tabla I).

Una vez aprobada la Ley, un análisis sobre el texto definitivo de la misma, centrado en las variaciones de puntuaciones realizadas sugiere un resultado quizás no tan radical pero similar. En el 
curso de la jornada sobre "Baremo de daños corporales. Nueva tabla VI", celebrada en Bilbao en enero de 2004, presentamos un análisis de estos cambios desde una perspectiva médica, en la que intentamos agrupar las secuelas que realmente sufren cambios de puntuación, aquellas realmente nuevas o las que desaparecen, considerando la necesidad de estimar estas de forma única en el caso de referirse a categorías diagnósticas comunes si bien con gradaciones diversas (p.ej., anquilosis de codo en posición funcional y no funcional frente a anquilosis de codo de $0-30^{\circ}, 30-75^{\circ}$ y $75-150^{\circ}$, consideradas como una categoría única de anquilosis) [8] (tabla 2)

\section{EXTREMIDAD SUPERIOR}

\begin{tabular}{|l|r|}
\hline Puntos globales de disminución & 147 \\
\hline Categorias que disminuyen puntuación & 33 \\
\hline Categorias que desaparecen & 26 \\
\hline Puntos globales de aumento & 21 \\
\hline Categorias que aumentan puntuación & 3 \\
\hline EXTREMIDAD INFERIOR \\
\hline Puntos globales de disminución & 437 \\
\hline Categorias que disminuyen puntuación & 60 \\
\hline Categorias que desaparecen & 8 \\
\hline Puntos globales de aumento & 32 \\
\hline Categorías que aumentan puntuación & 5 \\
\hline
\end{tabular}

Tabla 1: Análisis del Grupo Parlamentario Socialista durante la tramitación parlamentaria del Proyecto de Ley sobre los cambios de puntuación en la tabla VI de la Ley 30/1995 [9].

\begin{tabular}{|l|r|}
\hline EXTREMIDAD SUPERIOR \\
\hline Puntos globales de disminución & 81 \\
\hline Categorias que disminuyen puntuación & 21 \\
\hline Categorias que desaparecen & 31 \\
\hline Puntos globales de aumento & 39 \\
\hline Categorias que aumentan puntuación & 7 \\
\hline EXTREMIDAD INFERIOR \\
\hline Puntos globales de disminución & 117 \\
\hline Categorias que disminuyen puntuación & 22 \\
\hline Categorias que desaparecen & 26 \\
\hline Puntos globales de aumento & 57 \\
\hline Categorias que aumentan puntuación & 6 \\
\hline
\end{tabular}

Tabla 2: Análisis presentado en la Jornada sobre "baremo de daños corporales. Nueva tabla VI", celebrada en enero de 2004 en Bilbao sobre los cambios de puntuación en la tabla VI de la Ley 30/1995 [8].

De este modo, un análisis superficial y no riguroso de la nueva redacción del baremo nos llevaría a concluir que en la Ley 34/2003 se aprecian una serie de cambios:

I. Existe una desaparición masiva de categorías completas de secuelas (p. ej., atrofias musculares)

2. Existe una disminución global de los puntos por secuela

3. Se han reestructurado algunas categorías diagnósticas mediante métodos de agrupación (p. ej., deformidades del pie)

4. Existe una redefinición terminológica y conceptual de ciertas secuelas (p.ej. Arcos de movimiento, pseudoartrosis o anquilosis-artrodesis)

5. La redacción legal parece orientada a favorecer a las compañías de seguros, dado que estas pagarían por menos secuelas y una cantidad menor por secuela.

Sin embargo, como suele suceder, los análisis superficiales de sistemas complejos en ocasiones son acertados y en otras yerran en sus apreciaciones. Por ello, un análisis de un sistema complejo como es el propuesto en el baremo de la Ley 34/2003 exige un análisis más pormenorizado de sus características antes de estimar su idoneidad o imperfección.

\section{EL PROBLEMA DE LAS SECUELAS TEMPORALES:}

Durante el proceso de tramitación parlamentaria de la nueva Ley 34/2003, se incluyó entre las reglas generales de aplicación de la tabla la regla tercera relativa a la interpretación de las denominadas "secuelas temporales". La génesis de esta regla general ya ha sido debatida en múltiples foros. Los miembros del Comité de Expertos convocado por UNESPA para el seguimiento de la 
Ley 30/I 995 y autores de la nueva tabla de la Ley 34/2003 ya han indicado que dicha regla no ha sido producto de sus recomendaciones sino de fuentes ajenas al Comité $[5,6]$. En todo caso, la aplicación de la regla condiciona una serie de incongruencias conceptuales e indemnizatorias incompatibles con un baremo de indemnización de lesiones maduro.

Conceptualmente, el término "secuela temporal" definido como "aquellas (secuelas) Ilamadas a curarse a corto o medio plazo (...) después de haberse alcanzado la estabilización lesional" [ I ] resulta un término ajeno al espíritu general de los distintos sistemas de indemnización de secuelas en los países occidentales. Es admitido en todos los sistemas de indemnización de lesiones, tanto basados en sistemas abiertos como cerrados, que el término de secuela queda reservado para aquellos estados físicos o psíquicos previsiblemente permanentes según criterios médicos basados en la experiencia del médico valorador. Algunos sistemas, como el anglosajón, prevén el caso de eventuales mejorías en el estado físico o psíquico en algunos casos [l I], pero estas eventuales mejorías han de resultar parciales para ser indemnizables como secuelas. Cualquier otra circunstancia, en la que a corto o medio plazo pueda existir una mejoría en el estado físico o psíquico del lesionado y que pueda conducir a la completa desaparición del estado patológico del mismo debe ser considerada dentro del periodo de "curación" o de "estabilización" y según los términos de las leyes 34/2003 y 30/I995 evaluado en función del número de días que el proceso morboso tardará realmente en curar bajo asistencia facultativa en régimen hospitalario o no, diferenciando la existencia o no de incapacidad asociada para el desarrollo normal de sus actividades habituales o impedimento. Exigiría, por tanto, el seguimiento personalizado del lesionado hasta su curación o estabilización y nunca una estimación basada en cálculos "razonables", dado que el período de estabilización o de curación, pese a responder a unos plazos habitualmente enmarcados en unos términos previsibles, es absolutamente particular e individual. Parafraseando el clásico aforismo médico, actuar de otra forma sería olvidar que "no existen lesiones, sino lesionados". Por tanto, el término secuela temporal, tal y como es recogido en la regla general tercera del baremo, resulta un contrasentido conceptual desde el punto de vista técnico médico legal.

Por otra parte, la aplicación de la regla tercera, tal y como se expresa en el texto legal, exige que se indemnice la "secuela temporal" con arreglo a un cálculo basado en el "cálculo razonable de su duración". Dejando a un lado el absurdo conceptual del término, ya apuntado, y la dificultad de establecer los límites aceptables de lo razonable en una especialidad no basada a menudo en evidencias científicas, la aplicación estricta de esta regla puede conducir a auténticos absurdos indemnizatorios, como sería el de dar lugar a indemnizaciones superiores para "secuelas temporales" llamadas a curarse y de menor entidad clínica que para otras de mayor entidad clínica y previsiblemente definitivas. Esta situación resulta nuevamente incompatible con el adecuado principio de proporcionalidad que se le debe exigir a todo sistema de indemnización de lesiones, sea este basado en tablas, baremos o en cualquier otro medio de reparación.

La inclusión de la regla general tercera podría considerarse un loable intento por parte del legislador de suplir la deficiencia de categorías de secuelas permanentes que existe en la nueva redacción del baremo. Sin embargo, la solución propuesta carece del necesario rigor desde un punto de vista médico legal y puede obligar al perito médico forense a plantearse soluciones más adecuadas a las deficiencias del baremo, aun a costa de obviar una regla general de aplicación obligada por mandato legal. Estas soluciones deben estar destinadas a evitar absurdos indemnizatorios o ausencias de indemnización por la incapacidad del baremo para crear un marco abierto en el que todos los posibles estados clínicos con repercusión funcional real tengan cabida adecuada como secuelas permanentes indemnizables $[7,8]$. 


\section{PRINCIPIOS METODOLÓGICOS DE UN SISTEMA DE CUANTIFICACIÓN DE DAÑOS PERSONALES:}

En primer lugar, un sistema de indemnización de daños personales debe establecer de forma clara la modalidad de daño personal que busca indemnizar. De este modo, los sistemas destinados a ser aplicados por médicos legistas o médicos valoradores deben servir únicamente para la cuantificación de los daños físicos y psíquicos. En la medida de lo posible, la indemnización por daños morales, loss of amenities, future earnings o cualesquiera otras modalidades de daños personales debiera ser valorada de forma independiente y no por un perito médico, cuya cualificación profesional tal vez no sea la más adecuada para este tipo de valoraciones.

Todo baremo de cuantificación de daños personales físicos y psíquicos, especialmente si este está destinado a la reparación económica de los mismos, debiera disponer de varios elementos definitorios de su redacción:

I. Adecuado fundamento científico.

2. Marco abierto y no excluyente de asignación de estados clínicos que permitan la inclusión de todo tipo de estados clínicos con repercusión funcional real.

3. Redacción clara con adecuación terminológica a los términos manejados en la literatura médica actualizada.

4. Adecuada consistencia interna o validez en sus sistemas de asignación de grados de gravedad.

5. Congruencia con otros baremos ya existentes y validados, con estudios válidos que demuestren la eficacia de los métodos de cuantificación como predictores de un menoscabo funcional proporcionado y con las recomendaciones de los comités de expertos, cuando estos se hallen disponibles.

Estos principios, exigibles a toda prueba biomédica, resultan esenciales para obtener dos objetivos principales en la aplicación práctica de un baremo de daños corporales:

I. Una correcta asignación de gravedad relativa al estado clínico residual de un lesionado basada en un principio de proporcionalidad.

2. Una correcta reproducibilidad de los resultados en el caso de que dos peritos expertos evalúen de forma independiente al mismo lesionado.

Ambos objetivos permitirán que la asignación de la gravedad relativa del daño sea aceptable $y$, por tanto, se pueda realizar una adecuada reparación económica del mismo de acuerdo con los principios que el legislador establezca para la reparación de los daños causados.

En relación con el análisis de los cuatro primeros elementos, los dos trabajos subsiguientes analizarán más específicamente estos en relación con los capítulos de columna vertebral, extremidades superiores e inferiores y los síndromes psiquiátricos del nuevo baremo.

El quinto elemento se enfrenta con una realidad difícil de obviar y es la de que ninguno de los sistemas de baremo de daños personales existentes está exento de críticas respecto a su fundamentación científica y su consistencia interna. En realidad, la propia subespecialidad de la valoración del daño corporal es una rama de la medicina legal que, como la mayor parte de las áreas del quehacer médico forense, tiene un fundamento científico mal definido y no basado en evidencias científicas.

El baremo de aplicación más generalizado en los países occidentales, especialmente los de la esfera anglosajona, es la llamada tabla AMA (American Medical Association), elaborada por la Asociación Americana de Medicina. En nuestro país, una adaptación de la misma tabla fue realizada y publicada oficialmente en 1999 como sistema de valoración del grado de minusvalía por parte del Ministerio de Asuntos Sociales [10]. Sin embargo, han sido múltiples las críticas que ha recibido la edición más reciente de las tablas AMA tras su publicación. En Australia, son varios los estados que 
utilizan adaptaciones de la tabla americana original como medio de establecimiento del grado de menoscabo físico y psíquico tras accidentes de tráfico [l l ]. Este menoscabo, una vez cuantificado, es informado a las autoridades pertinentes para, sobre la base de este cálculo, establecer de forma individualizada la cuantía de indemnización. De forma análoga, países como Canadá o USA utilizan en muchos de sus estados la tabla original como medio para establecer el grado de menoscabo corporal tras un accidente de tráfico.

Pese a las múltiples bondades de su última edición como método de cuantificación genérica del menoscabo, esta tabla ha sido criticada de forma severa en múltiples trabajos en la bibliografía internacional [12]. Se le ha criticado a la tabla AMA, entre otros aspectos, su falta de fundamento en estudios previos basados en evidencias de los criterios de cuantificación del porcentaje de menoscabo estimado [13]. En particular, se ha destacado la eficacia dudosa de los criterios propuestos en el segmento de tobillo y pie frente a otros criterios de cuantificación del daño corporal [13] y la especial dificultad de valorar el dolor como factor limitante funcional. Del mismo modo, se ha puesto en tela de juicio la validez de la cuantificación del menoscabo en la región de columna vertebral basado en estimaciones de arcos de movilidad $[14,15,16]$ o la validez del método AMA para estimar los daños leves a moderados en la extremidad superior [17]. En la misma línea, se ha criticado la validez del método AMA como predictor del menoscabo real en visión binocular, especialmente cuando la diferencia de agudeza visual entre ambos ojos es significativa $[18,19]$. Finalmente, se ha criticado severamente la reproducibilidad de las estimaciones en el caso del daño psicológico y se han propuesto algunos métodos alternativos y complementarios al método AMA para mejorar su reproducibilidad, como los apuntados por Epstein et al e incluidos en el sistema de la MAA (Motor Accidents Authority) de New South Wales, en Australia [ I I, I 2].

Por otra parte, una comparación entre la tabla AMA, como referente de tabla consensuada y aceptada pese a todas sus imprecisiones y a su fundamento discutible, y la tabla-baremo española puede resultar un ejercicio de malabarismo conceptual. Tal y como se ha explicado, la doctrina jurisprudencial en países de la esfera anglosajona (sistema cerrado) es distinta de la de los países que aplican un sistema abierto de reparación de daños personales. Algunos autores nacionales, de hecho, han querido extrapolar las conclusiones de estudios como los de Cassidy et al en Canadá [20] para aplicarlos al contexto español. Este estudio especificaba la radical disminución de las demandas de indemnización por daños personales en Canadá tras la eliminación de la reparación por sufrimiento y daños morales en aquellos casos que no alcanzaban un nivel mínimo establecido de menoscabo funcional. De forma análoga, se ha actuado en países como Australia, donde las iniciativas normativas han seguido una guía similar. Este tipo de extrapolación resulta dudoso dadas las diferencias ya señaladas anteriormente del Derecho español frente al Derecho en los países anglosajones.

Además, la propia redacción del baremo de la Ley 30/1995 y de la Ley 34/2003 presenta un escollo insalvable en este sentido. Así, la tabla-baremo española presenta la peculiaridad de que los daños cuantificados en la tabla $\mathrm{VI}$ por rangos de puntuación agrupan en las mismas categorías tanto a los daños corporales como a los daños morales con independencia de factores personales del lesionado (anexo, norma $1^{\circ}$, punto 7 de la Ley 30/I995 [2]). Esta asociación de términos de indemnización resulta útil para el legislador al agrupar conceptos indemnizatorios diversos en un sistema de cuantificación único, pero resulta en realidad un obstáculo para el médico valorador, quien quizás no sea el profesional más adecuado para valorar los daños morales, sino sólo los menoscabos funcionales. 
Por el contrario, las aplicaciones de las tablas AMA en sus distintas versiones internacionales incluyen únicamente un sistema de cuantificación del grado porcentual de menoscabo genérico inducido al lesionado. Esta valoración porcentual sólo sirve al jurista como punto de partida para establecer la indemnización individualizada, en función de las características del sujeto y por daños específicos por sufrimiento y dolor y loss of amenities.

Por supuesto, el sistema anglosajón permite la adaptación de los resultados de menoscabo genérico estimado por la tabla AMA a las condiciones particulares del lesionado. Se remarca en el preámbulo de la tabla americana la diferencia conceptual entre menoscabo genérico (impairment), discapacidad (disability) y minusvalía (handicap) y la congruencia de estas diferenciaciones con las recomendaciones de la OMS (Organización Mundial de la Salud). El ejemplo más conocido y recogido en la propia norma de aplicación de las tablas AMA, es el de que el menoscabo genérico por lesión en los dedos de la mano, único e independiente de la actividad del sujeto, no conlleva el mismo grado de discapacidad o de minusvalía en un bibliotecario o en un pianista profesional. De este modo, en Australia las guías MAA consideran que la estimación final del perito médico debe realizarse bajo la forma de una estimación del porcentaje de menoscabo genérico con acotaciones relativas a la posible discapacidad, pero nunca estimaciones sobre minusvalía. Esta última, debe ser estimada por el Tribunal juzgador al implicar a la forma específica en la que el menoscabo y la discapacidad afectan a las actividades efectivas habituales del sujeto. De este modo, la indemnización final se acompañará de una reparación independiente por los loss of ammenities y por los future earnings estimada libremente por el juez.

Estas circunstancias en nuestro ordenamiento jurídico, encasillado en la aplicación estricta de las distintas tablas de las leyes 30/1995 y 34/2003, no son posibles en la generalidad de lesionados. En realidad, los principios conceptuales sobre los que se fundamentan las tablas AMA y sus adaptaciones internacionales para la reparación del daño corporal y los principios de la tabla baremo europea responden a orientaciones doctrinales radicalmente distintas y se enmarcan en contextos jurídicos con grandes similitudes pero con diferencias significativas.

Sin embargo, a falta de otros referentes, cualquier comparación de validez que se deba realizar sobre el desarrollo de una sistema de tabla-baremo de indemnización de daños personales es conveniente realizarla con la tabla AMA. Pese a todas las críticas a sus fundamentos esta tabla conforma el sistema de cuantificación de menoscabo funcional más generalizado en los países industrializados $y$, al tiempo, presenta algunas características de validez que la hacen un referente tal vez mejorable pero necesario para otros sistemas de baremo.

En el contexto europeo, en mayo de 2003 también se culminó el proyecto de una nueva "guía baremo europea para la evaluación de las lesiones físicas y psíquicas" [2I]. Este proyecto está pendiente de revisión por parte del Parlamento Europeo antes de su publicación oficial como recomendación comunitaria de obligado cumplimiento en los países de la Unión. El proyecto fue presentado por los miembros de CEREDOC (Confederación Europea de Expertos en Evaluación y Reparación del Daño Corporal) y su grupo de trabajo presenta una distribución de miembros por países de un 22\% de Bélgica, II\% de Portugal, II\% de Francia, II\% de Italia, I I\% de Alemania y 33\% de España. Además, de los tres españoles miembros del grupo de trabajo europeo, dos de ellos han sido miembros del grupo de trabajo de UNESPA que ha elaborado la tabla baremo española de la Ley 34/2003 [5]. En cuanto al grupo de médicos consultados para la elaboración de la tabla europea, destaca el hecho de que sólo constan médicos especialistas procedentes de España, Bélgica, Francia, Portugal e Italia, no existiendo representación del resto de países miembros de la Unión. 
El proyecto europeo "guía baremo europea para la evaluación de las lesiones físicas y psíquicas" presenta, como era previsible, grandes similitudes en su estructuración y su fundamento científico con el sistema español pero, curiosamente, también algunas diferencias significativas que afectan a gran parte del baremo. Estas diferencias hacen a la tabla baremo europea un sistema más preciso en muchos aspectos. Sin embargo, al tiempo, en la tabla europea se repiten errores conceptuales significativos. De cualquier manera, aún es pronto para elaborar conclusiones de ningún tipo sobre esta nueva propuesta. Posiblemente, durante el proceso de tramitación en el Parlamento Europeo se discutan aspectos sustanciales del proyecto en los que los puntos de vista de los países no consultados previamente por la CEREDOC sobre la cuestión serán sin duda altamente enriquecedores.

\section{BIBLIOGRAFÍA:}

I. Ley 34/2003 de 4 de noviembre de modificación y adaptación a la normativa comunitaria de la legislación de seguros privados. BOE 5-II-2003. http://www.justizia.net/docuteca/ficheros.asp?intcodigo $=|58| \& \mid d D o c=S P$ 2. Ley 30/1995 de 8 de noviembre de ordenación y supervisión de seguros privados. BOE 9-II-1995, pp.32480-32567.

3. Vicente Domingo E. Los daños corporales: tipología y valoración. JM Bosch Editor SA. 1994.

4. Miralles RC. Valoración del daño corporal en el aparato locomotor. Masson. 2001.

5. Borobia C. Análisis del anteproyecto de la reforma de la tabla VI de la Ley 30/1995. VII Jornadas Catalanas de Actualización en Medicina Forense. Barcelona 12-6-2003.

6. Alonso J. La nueva tabla VI. Presentación y análisis. Principales mejoras y diferencias. Jornada sobre "baremo de daños corporales. Nueva tabla VI", Bilbao 26-I-2004. http://www.justizia.net/ivml 7. Portero $G$. Valoración de la tabla psiquiátrica. Comentarios y críticas a la Ley 34/2003. Jornada sobre "baremo de daños corporales. Nueva tabla VI", Bilbao 26-I-2004. http://www.justizia.net/ivml 8. Garamendi PM. Cambios realizados en la tabla VI de la Ley 30/1995 por la Ley 34/2003. Extremidades superiores e inferiores. Jornada sobre "baremo de daños corporales. Nueva tabla VI", Bilbao 26-I2004. http://www.justizia.net/ivml

9. Diario de sesiones del Congreso de los Diputados. Pleno y Diputación permanente. Sesión plenaria número 258 (sesión extraordinaria) celebrada el 8-7-2003. 13958-68. http://www.congreso.es

10. Real Decreto 1971/1999, de 23 de diciembre de procedimiento para el reconocimiento, declaración y calificación del grado de minusvalía. BOE 26-I-2000, 3317-34I0. http://www.boe.es

II. New South Wales Motor Accidents Authority Guidelines for the Assessment of Permanent Impairment of a Person injured as a result of a motor accident. MAA Guidelines, March 2000. http://www.maa.nsw.gov.au

12. Rondinelli RD, Katz RT. Merits and shortcomings of the American Medical Association Guides to the Evaluation of Permanent Impairment, 5th edition. A physiatric perspective. Phys Med Rehabil Clin N Am. 2002 May; 13(2): 355-70, x. http://www.ncbi.nlm.nih.gov/entrez/query.fcgi?cmd = Retrieve\&db $=$ pubmed\&dopt $=$ Abstract\&list_uids $=|2| 2285 \mid$
13. Harper JD. Determining foot and ankle impairments by the AMA fifth edition guides. Foot Ankle Clin. 2002 Jun; 7(2): 29I-303. http://www.ncbi.nlm.nih.gov/entrez/query.fcgi?cmd = Retrieve\&db $=$ pubmed\&dopt $=$ Abstract\&list_uids $=12462 \mathrm{III}$

14. Zuberbier OA, Hunt DG, Kozlowski AJ, Berkowitz J, Schultz IZ, Crook JM, Milner RA. Commentary on the American Medical Association guides' lumbar impairment validity checks. Spine. 2001 Dec 15; 26(24): 2735-7. http://www.ncbi.nlm.nih.gov/entrez/query.fcgi?cmd = Retrieve\&db $=$ pubmed\&dopt $=$ Abstract\&list_uids $=11740365$

15. Nattrass CL, Nitschke JE, Disler PB, Chou MJ, Ooi KT. Lumbar spine range of motion as a measure of physical and functional impairment: an investigation of validity. Clin Rehabil. 1999 Jun; 13(3): 21I-8. http://www.ncbi.nlm.nih.gov/entrez/query.fcgi?cmd = Retrieve\&db $=$ pubmed\&dopt $=$ Abstract\&list uids $=10392648$

16. Lowery WD Jr, Horn TJ, Boden SD, Wiesel SW. Impairment evaluation based on spinal range of motion in normal subjects. J Spinal Disord. 1992 Dec; 5(4): 398-402. http://www.ncbi.nlm.nih.gov/entrez/query.fcgi!cmd= Retrieve\&db $=$ pubmed\&dopt $=$ Abstract\&list_uids $=1490036$

17. Rondinelli RD, Dunn W, Hassanein KM, Keesling CA, Meredith SC, Schulz TL, Lawrence NJ. A simulation of hand impairments: effects on upper extremity function and implications toward medical impairment rating and disability determination. Arch Phys Med Rehabil. 1997 Dec; 78(I2): 1358-63. http://www.ncbi.nlm.nih.gov/entrez/query.fcgi?cmd = Retrieve\&db $=$ pubmed\&dopt $=$ Abstract\&list_uids $=9421991$

18. Massof RW. The measurement of vision disability. Optom Vis Sci. 2002 Aug; 79(8): 516-52. http://www.ncbi.nlm.nih.gov/entrez/query.fcgi?.cmd = Retrieve\&db $=$ pubmed\&dopt $=$ Abstract\&list_uids $=12199545$

19. Rubin GS, Munoz B, Bandeen-Roche K, West SK. Monocular versus binocular visual acuity as measures of vision impairment and predictors of visual disability. Invest Ophthalmol Vis Sci. 2000 0ct; 4I(II): 3327-34. http://www.iovs.org/cgi/content/full/4l//I/3327

20. Cassidy JD, Carroll LJ, Cote P, Lemstra M et al. "Effects of eliminating compensation for pain and suffering on the outcome of insurance claims for whiplash injury". N Engl J Med, 2000;342(16): II79-II86. http://www.ncbi.nlm.nih.gov/entrez/query.fcgi?cmd = Retrieve\&db $=$ pubmed\&dopt $=$ Abstract\&list_uids $=10770984$

21. Guide barème européen d'évaluation des atteintes à l'intégrité physique et psychique. Groupe Rothley. 25-mai-2003. http://la-plaza.com/vdc/BD/doc/revisiones/index.html 